\title{
Erratum to: Electric field effects on nuclear spin-spin coupling tensors and chiral discrimination via NMR spectroscopy
}

Gabriel I. Pagola $\cdot$ Marta B. Ferraro •

Stefano Pelloni $\cdot$ Paolo Lazzeretti $\cdot$ Stephan P. A. Sauer

Published online: 12 August 2011

(C) Springer-Verlag 2011

Erratum to: Theor Chem Acc (2011) 129:359-366

DOI 10.1007/s00214-010-0851-2

Figure 1 contains numbering errors for the nuclei of $\left(\mathrm{R}_{\mathrm{a}}\right)$-1,3-dimethylallene. The correct figure is reported here.
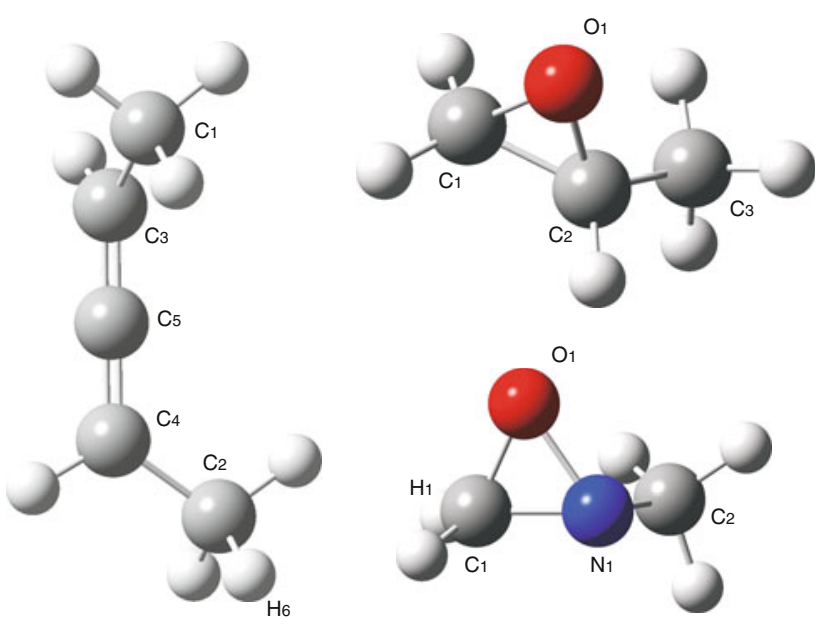

Fig. 1 ( $\mathrm{R}_{\mathrm{a}}$ )-1,3-dimethylallene, (2R)-2-methyloxirane, and (2R)- $\mathrm{N}$ methyloxaziridine

The online version of the original article can be found under doi:10.1007/s00214-010-0851-2.

G. I. Pagola - M. B. Ferraro

Departamento de Física, Facultad de Ciencias Exactas y

Naturales, Universidad de Buenos Aires and IFIBA,

CONICET Ciudad Universitaria, Pabellon 1,

1428 Buenos Aires, Argentina

S. Pelloni · P. Lazzeretti ( $\square)$

Dipartimento di Chimica dell'Università degli Studi di Modena

e Reggio Emilia, Via Campi 183, 41124 Modena, Italy

e-mail: lazzeret@unimore.it

S. P. A. Sauer

Department of Chemistry, University of Copenhagen,

Universitetsparken 5, 2100 Copenhagen, Denmark 